\title{
Context affects implicit learning of spatial bias depending on task relevance
}

\author{
Injae Hong ${ }^{1} \cdot$ Su Keun Jeong ${ }^{2} \cdot$ Min-Shik Kim ${ }^{1}$ \\ Published online: 6 January 2020 \\ (C) The Psychonomic Society, Inc. 2020
}

\begin{abstract}
Recent studies on the probability cueing effect have shown that a spatial bias emerges toward a location where a target frequently appears. In the present study, we explored whether such spatial bias can be flexibly shifted when the target-frequent location changes depending on the given context. In four consecutive experiments, participants performed a visual search task within two distinct contexts that predicted the visual quadrant that was more likely to contain a target. We found that spatial attention was equally biased toward two target-frequent quadrants, regardless of context (context-independent spatial bias), when the context information was not mandatory for accurate visual search. Conversely, when the context became critical for the visual search task, the spatial bias shifted significantly more to the target-frequent quadrant predicted by the given context (context-specific spatial bias). These results show that the task relevance of context determines whether probabilistic knowledge can be learned flexibly in a context-specific manner.
\end{abstract}

Keywords Probability cueing $\cdot$ Context $\cdot$ Implicit learning $\cdot$ Task relevance $\cdot$ Incidental learning

Statistical regularities exist in the real world, and learning these rules, whether explicitly or implicitly, can be advantageous. For example, knowing where prey frequently appears is critical for a predator to be successful in its hunt. Previous studies have used a probability cueing paradigm to investigate the learning of location probability (Druker \& Anderson, 2010; Geng \& Behrmann, 2002, 2005; Jiang, Swallow, Rosenbaum, \& Herzig, 2013c). In a typical probability cueing experiment, participants perform a visual search task to find a target among distractors, while the target is more likely to appear in a certain location than in other locations. The more frequent target location is called a "rich" location, whereas the less frequent target location is called a "sparse" location. As the trials progress, the target detection times become faster when the target is presented in a rich location. This location probability learning usually occurs gradually, without conscious awareness or an intention to learn (Jiang, 2018; Jiang, Sha, \& Sisk, 2018; Jiang \& Sisk, 2019; Jiang, Swallow, \& Rosenbaum, 2013b).

Min-Shik Kim

kimm@yonsei.ac.kr

1 Department of Psychology, Yonsei University, Seoul, South Korea

2 Cognitive Science Research Group, Korea Brain Research Institute, Daegu, South Korea
In a real-life situation, a frequent target location may differ depending on the given situation. Imagine the case of a stressed graduate student: She usually stays in the laboratory during the day, but frequently she drops by a nearby pub at night. If you repeatedly encounter her at the laboratory during the day, and at a nearby pub at night, you could easily determine where to go first to find her at 10:00 p.m. Likewise, the probability of the target location can differ depending on the "context." It would be beneficial to learn the context-specific probabilistic distribution and to shift spatial attention to a probable target location that the given context predicts. However, little is known about whether implicit knowledge of the context can be learned and can guide spatial attention to a frequently predicted target location.

Several studies have speculated about the possibility of implicit learning of context information. For example, participants were able to learn that a location cue predicted the proportion of congruency, even though they were not consciously aware of the rule (Crump, Gong, \& Milliken, 2006; Hübner \& Mishra, 2016; Wendt, Kluwe, \& Vietze, 2008). In contextual cueing studies, the configuration of search distractors guided attention to a possible target location during visual search (Chun \& Jiang, 1998; Jiang \& Song, 2005; Kim \& Kim, 2016). Specifically, in a study by Jiang and Song, participants were able to learn distinctive sets of statistical regularities in two different contexts. 
In contrast, some studies have shown that context information failed to guide spatial attention, resulting in a contextindependent spatial bias. In Won and Jiang (2015), participants performed a visual search task with or without visual working memory load. Two distinct quadrants were assigned as rich quadrants, depending on whether or not there was a working memory load. However, the participants in Won and Jiang's study failed to associate that working memory load predicted the target-rich quadrant, instead showing a context-independent spatial bias. In another study, when two types of targets were presented during a contextual cueing paradigm, participants did not associate the target identity with the distractor configurations (Ahn \& Kim, 2017). In other words, the target identity did not serve as a contextual cue that distinguished the spatial configurations.

One possible factor that could resolve these discrepancies regarding contextual guidance of spatial attention is the degree of task relevance. In Crump et al. (2006), shape cues predicted the congruency proportion of the Stroop task, but participants failed to use the shape-specific congruency information. However, other research (Crump, Vaquero, \& Milliken, 2008) showed successful learning of shape-specific knowledge about the congruency proportion when participants had to count how many times a certain shape cue was presented. The difference between these two studies can be explained by the different extents of attentional allocation to the shape cue. In other words, context-specific learning could occur when the context was actively attended and processed (Frensch \& Rünger, 2003).

In the present study, we aimed to investigate whether context information can be learned in order to bias spatial attention in an implicit manner, and whether the learning of such a spatial bias depends on the task relevance of the context information. In four experiments, participants were asked to search for a target among multiple distractors. The context was defined as either the color of the stimuli (Exps. 1, 2, and 3) or both the color of stimuli and the response dimension (Exp. 4), and the context predicted which quadrant of the display was more likely to contain a target. We predicted the presence of equal spatial biases toward the two target-frequent quadrants, regardless of the context (i.e., context-independent spatial bias), when the context information had low task relevance (Exps. 1 and 2), as opposed to spatial biases toward different quadrants that would depend on the context (i.e., context-specific spatial bias) when the context information was essential to the visual search task (Exps. 3 and 4).

\section{Experiment 1}

Experiment 1 was conducted to find out whether statistical information can be learned separately according to the context. Here the colors of the stimuli were defined as the context that contained a heterogeneous probabilistic distribution. The task was to find the target among the distractors, regardless of the color of the items.

\section{Method}

Participants A total of 38 participants were recruited for the experiment, for course credit or monetary reward. Thirty-six of the participants were recruited first, and two participants were later recruited after the exclusion of two participants who reported having noticed the rule (see the Procedure section), to ensure that the conclusion of this experiment was driven by implicit learning. All participants provided written consent before the experiment began. All of them reported normal or corrected-to-normal visual acuity and normal color vision. None of the participants were aware of the purpose of the experiment beforehand.

We defined the sample size using $G^{*}$ Power (Faul, Erdfelder, Lang, \& Buchner, 2007), on the basis of the effect size of a probability cueing study of similar design (Won \& Jiang, 2015). The effect size from the uneven probability distribution yielded 24 as an appropriate sample size. We increased this to 36 , a multiple of 12 , in order to counterbalance the rich quadrants by context, because a smaller spatial bias was expected when the target distribution became even in the testing phase. The same sample size was adjusted in all the remaining experiments.

Stimuli and apparatus An IBM-compatible computer and a 24-in. LED monitor were used. The resolution of the monitor was $1,920 \times 1,080$. The experiment was programmed using MATLAB with the Psychophysics Toolbox (Brainard, 1997; Pelli, 1997). All stimuli were presented on a gray background (RGB: $128,128,128)$. The search array contained one target $\mathrm{T}$, rotated $90^{\circ}$ to left or right, and 15 distractors of rotated Ls $\left(0^{\circ}, 90^{\circ}, 180^{\circ}\right.$, or $\left.270^{\circ}\right)$. The size of each stimulus was $0.82^{\circ} \times$ $0.80^{\circ}$. The items were colored either black (RGB: $0,0,0$ ) or white (RGB: 255, 255, 255), and all items in an array possessed an identical color. A red cross (RGB: 255, 0, 0) was used as a fixation. The display was divided into a $10 \times 10$ invisible matrix, with the size of each cell subtending $1.63^{\circ} \times$ $1.60^{\circ}$. The cells were allocated to quadrants, each quadrant containing 25 cells. Sixteen cells were randomly selected as item locations for every trial, so that location repetition rarely happened (Jiang, Won, \& Swallow, 2014b), with the constraint that equal numbers of stimuli were present in every quadrant. Within each cell, the item positions were randomly jittered horizontally and vertically within a range of $\pm 0.27^{\circ}$ from the center.

Procedure Participants were given 32 practice trials. The main experiment was composed of ten blocks: the training phase for the first eight blocks, and the testing phase for the remaining two blocks. Each block included 48 trials, for a total of 480 trials for the entire experiment, which lasted for about an hour.

On each trial, a fixation cross was presented for $500 \mathrm{~ms}$, followed by a search array that contained 16 items. 
Participants searched for a target (T) among distractors (L) and reported whether the target was tilted to the left or right. They were instructed to respond as quickly and accurately as possible. If an incorrect answer was reported, an error sound was given as feedback. The next trial began after an intertrial interval of $500 \mathrm{~ms}$. The presentation of the items in black or white was the context information in the present experiment; the number of trials with a black context was equal to that with a white context. The white-context and black-context trials were randomly intermixed within a block.

During the training phase, one quadrant was assigned to be the rich quadrant for each context, and the remaining three became the sparse quadrants. The rich quadrant contained a target in $50 \%$ of the trials, as opposed to $16.67 \%$ of the trials for the sparse quadrants. The two contexts were not associated with the same rich quadrant, and the locations of the rich quadrants were counterbalanced across all participants.

During the testing phase, a target appeared in all four quadrants equally often (i.e., 25\%) in both contexts. The testing phase was conducted in order to dissociate the effect of probability cueing from the repetitive shift of attention to the target location of high probability.

In the valid-rich condition, a target appeared in a rich quadrant predicted by the context of the given trial. In the invalid-rich condition, the rich quadrant of the other context contained a target. In the neutral-sparse condition, the target appeared in one of the two quadrants that neither context predicted as a target-frequent quadrant. A schematic description of the design and the statistical manipulation is shown in Fig. 1.
After the search task, participants were asked whether they felt that targets were presented in every quadrant with equal probabilities. Then they were informed of the rule that the black $T$ and the white $T$ appeared frequently in different quadrants. The participants were subsequently asked to guess the quadrant where the white $T$ and the black $T$ frequently appeared. The participants who answered that targets were presented without equal probabilities in all quadrants and correctly pointed to the rich quadrants for both the white-T and black-T contexts were regarded as having explicit knowledge of the probability manipulation, and thus were excluded from the analysis.

\section{Results}

JASP 0.9.1 (JASP Team, 2018) was used to analyze the reaction times (RTs). Trials with RTs exceeding $10 \mathrm{~s}$ were removed from the analysis (Jiang, Swallow, Won, Cistera, \& Rosenbaum, 2015; Won \& Jiang, 2015), resulting in elimination of $2.33 \%$ of the trials. Accuracy reached $98.70 \%(S D=1.26 \%)$. RTs for correctly answered trials were analyzed.

Typical probability cueing studies predict that RTs would be faster when the target appeared in the rich quadrants than when the target appeared in the sparse quadrants. Therefore, the probability cueing effect was measured by comparing the RTs in the neutral-sparse condition with the RTs in the valid-rich and the invalid-rich conditions.

To find out the context effect, it is critical to compare the search performance between the valid-rich and the invalid-rich conditions. If participants learned the separate sets of statistical (a)

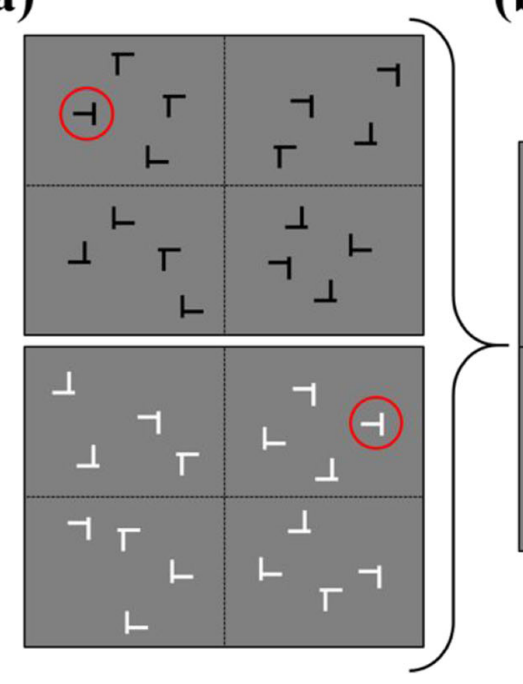

Fig. 1 Schematic description of Experiment 1. Items are not drawn to scale, and the quadrant borders were not shown in the experiment. (a) Example displays of a black context and a white context. The circles on the targets were not shown in the experiment. (b) In this example, the upper-left quadrant is the black rich quadrant, and the upper-right quadrant is the white rich quadrant. The remaining quadrants are the sparse (c)

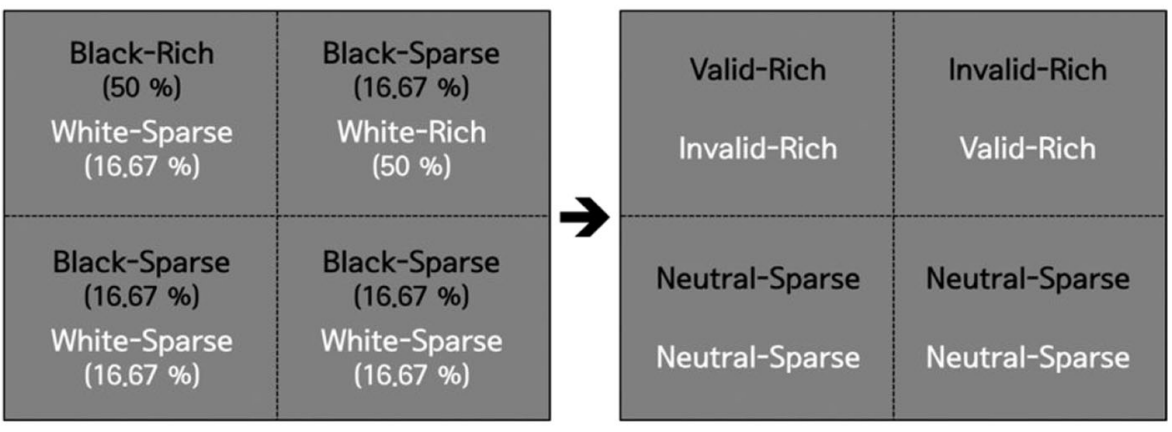

quadrants. (c) In the black context, the upper-left quadrant becomes a "valid-rich" quadrant, and the upper-right quadrant becomes an "invalid-rich" quadrant. Likewise, in the white context, the upper-right quadrant is the "valid-rich" quadrant, and the upper-left quadrant is the "invalid-rich" quadrant. The other two sparse quadrants are "neutral-sparse" quadrants. 
knowledge by context, then the RTs would be faster when a target appeared in the valid-rich quadrant than when it appeared in the invalid-rich quadrant, showing a context-specific spatial bias. In contrast, if participants failed to learn context-specific statistical knowledge, then no difference in the RTs would be observed between when a target appeared in the valid-rich and invalid-rich quadrants, showing a context-independent spatial bias.

Training phase Search performance by probability (valid-rich, invalid-rich, neutral-sparse) and block (1-8) was analyzed using a repeated measures analysis of variance (ANOVA) to examine probability learning over time (see Fig. 2). We performed a Bayesian analysis as well as a frequentist analysis, to quantify the evidence for each of the factors (Wagenmakers, Love, et al., 2018a; Wagenmakers, Marsman, et al., 2018b). A GreenhouseGeisser correction was applied in every frequentist analysis when the sphericity assumption was violated. A significant main effect of probability was observed, $F(1.367,47.853)=6.646, p=.007$, $\eta^{2}=.160, \mathrm{BF}_{10}=3,731.844$. Each level of the probability factor was contrasted in pairs to discover the probability cueing effect (contrast between the neutral-sparse and valid-rich conditions and between the neutral-sparse and invalid-rich conditions) and the context effect (contrast between the valid-rich and invalid-rich conditions). The results showed a significant probability cueing effect, in which the RTs of the valid-rich $(M$ $=2,962.55 \mathrm{~ms}, S D=528.16 \mathrm{~ms})$ and the invalid-rich $(M=$ $3,031.11 \mathrm{~ms}, S D=479.19 \mathrm{~ms}$ ) conditions were significantly faster than the RTs of the neutral-sparse $(M=3,217.72 \mathrm{~ms}, S D$ $=485.91 \mathrm{~ms})$ condition, $F(1,35)=9.905, p=.003, \mathrm{BF}_{10}=$ $1.235 \mathrm{e}+06$, and $F(1,35)=4.513, p=.041, \mathrm{BF}_{10}=12.887$, respectively. However, a context effect was not observed, $F(1$, $35)=3.199, p=.082, \mathrm{BF}_{01}=4.438$. Search times decreased as blocks continued, $F(7,245)=17.665, p<.001, \eta^{2}=.335, \mathrm{BF}_{10}=$ $2.933 \mathrm{e}+17$, which can be interpreted as a practice effect. The interaction between probability and block was not significant, $F(14,190)=1.144, p=.316, \eta^{2}=.032, \mathrm{BF}_{01}=196.697$.

Testing phase The testing phase, in which the target appeared in the four quadrants with equal probabilities, was performed to find out whether the probability learning was context-specific or context-independent. A repeated measures ANOVA with probability (valid-rich, invalid-rich, neutral-sparse) and block (9 and 10) as factors yielded a significant main effect of probability, $F(2,70)=$ $3.509, p=.035, \eta^{2}=.091, \mathrm{BF}_{10}=2.348$. A probability cueing effect was revealed, in which the RTs in the valid-rich $(M=$ $2,704.28 \mathrm{~ms}, S D=631.00 \mathrm{~ms})$ and the invalid-rich $(M=$ $2,708.37 \mathrm{~ms}, S D=655.21 \mathrm{~ms}$ ) conditions were faster than the RTs in the neutral-sparse $(M=2,906.93 \mathrm{~ms}, S D=565.59 \mathrm{~ms})$ condition, $F(1,35)=4.707, p=.037, \mathrm{BF}_{10}=2.481$, and $F(1,35)$ $=4.906, p=.033, \mathrm{BF}_{10}=3.353$, respectively. However, a context effect was not found, when comparing the RTs of the valid-rich and invalid-rich conditions, $F(1,35)<0.001, p=.985, \mathrm{BF}_{01}=$ 7.717, showing moderate evidence for a context-independent spatial bias. The main effect of block and the interaction between probability and block were not statistically significant, $F(1,35)=$ $0.001, p=.970, \eta^{2}=.000, \mathrm{BF}_{01}=6.587 ; F(2,70)=0.035, p=$ $.965, \eta^{2}=.001, \mathrm{BF}_{01}=11.011$.

Some participants $(N=24)$ were assigned to two adjacent rich quadrants (e.g., the first and second quadrants), and some others $(N=12)$ were assigned to two diagonal rich quadrants (e.g., the first and third quadrants). Two adjacent rich quadrants and two adjacent sparse quadrants can be grouped as either rich or sparse, attenuating any possible location probability learning. If the adjacent configuration yielded grouping of the rich quadrants, a context-independent spatial bias should be observed for those with an adjacent configuration, and a context-specific spatial bias for those with a diagonal configuration. We split the participants according to the configuration of the rich or sparse quadrants and ran a repeated (a)

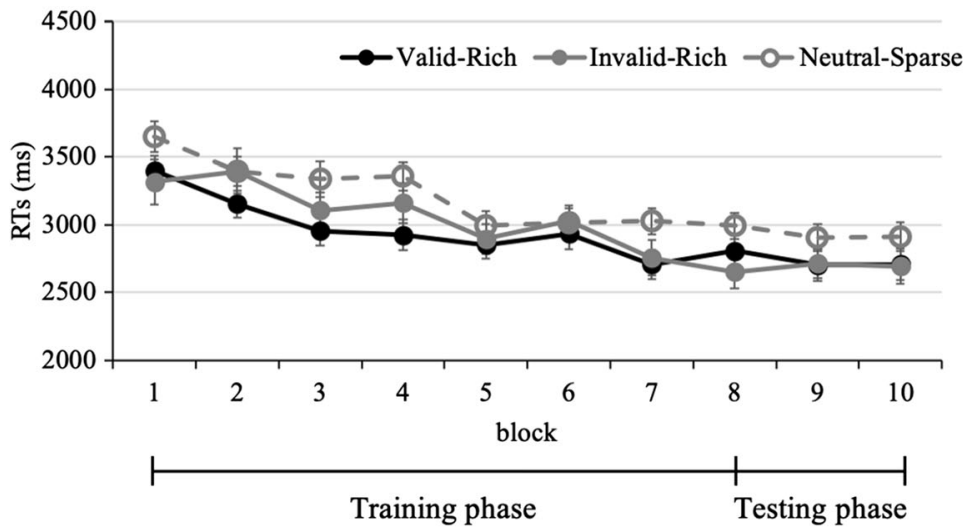

\section{(b)}

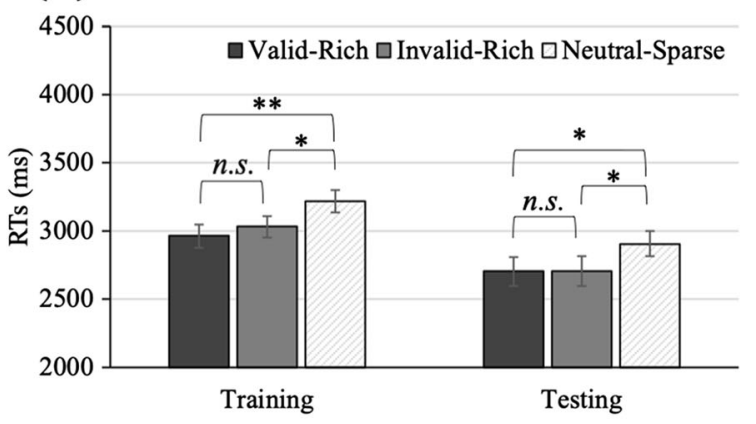

Fig. 2 Results of Experiment 1. (a) Reaction times (RTs) of each probability condition by blocks. The training phase was from Block 1 to Block 8, and the testing phase was in Blocks 9 and 10. (b) RTs by probability and phase in Experiment 1 . Error bars represent standard errors. ${ }^{*} p<.05,{ }^{* * *} p<.01$. 
measures ANOVA, newly including configuration (adjacent, diagonal) as a between-subjects factor. The interaction of probability and configuration was not significant, $F(2,68)=$ $1.795, p=.174, \eta^{2}=.010, \mathrm{BF}_{01}=1.61$, nor was the three-way interaction of probability, block, and configuration, $F(2,68)=$ $0.160, p=.852, \eta^{2}=.000, \mathrm{BF}_{01}=9.91$. The contextindependent spatial bias was not a weakened form of context-specific bias.

There was another possibility, that the context-independent spatial bias could have been driven by a leftward bias, which has been proven to dilute or affect the contextual cueing effect (Olson \& Chun, 2002). We separated out the 12 sets whose rich quadrants were both in the left hemifield $(N=6)$ or the right hemifield $(N=6)$. We conducted a 3 (probability: valid-rich, invalid-rich, neutral-sparse) $\times 2$ (block: 9,10$) \times 2$ (hemifield: left, right) repeated measures ANOVA to test for a leftward bias. However, the cueing effects did not differ across hemifields. We were not able to find any evidence of a leftward bias, $F(1,10)=$ $0.035, p=.856, \eta^{2}=.002, \mathrm{BF}_{01}=1.835$, nor of an influence of leftward bias on the spatial bias: Probability $\times$ hemifield interaction, $F(2,20)=0.045, p=.956, \eta^{2}=.001, \mathrm{BF}_{01}=4.980$.

\section{Discussion}

In Experiment 1, even though the general statistical property was learned and used to enhance search performance, knowledge of the probabilities was not distinguished between the two different contexts. When all trials from both contexts were combined, the two rich quadrants contained a target $33.33 \%$ of the time, and the two sparse quadrants contained a target $16.67 \%$ of the time. Search times were faster when a target appeared in one of the two rich quadrants, indicating that the net probability was acquired independently of the context.

This shows that context information is not attained implicitly even when the context contains useful information for the visual search task, displaying a context-independent spatial bias.

\section{Experiment 2}

In Experiment 1, a context-specific spatial bias was not found, even though participants did implicitly learn the overall probability distribution throughout the experiment. However, possibly the way that the search array was presented influenced implicit learning of the context-independent statistical information. In Experiment 1, context information was available to participants after a visual search array had been presented. Since an attentional shift to a target-frequent location could begin as soon as the onset of the search array (Jiang, Won, \& Swallow, 2014b; Won, Lee, \& Jiang, 2015), the shift of attention might have preceded the formation of a mental representation of the context, resulting in a context-independent spatial bias.
Experiment 2 was designed to provide context information prior to the search procedure, with the expectation that top-down attention would be engaged in order to categorize each incident into the appropriate context, and thus form separate knowledge of statistical distribution for each context. We examined the possibility of an implicit context-specific spatial bias when enough time was guaranteed for top-down attention to be addressed, through the use of a set of precues.

\section{Method}

Participants Thirty-eight new participants were recruited for course credit or monetary reward. As had also been done in Experiment 1, after recruiting 36 participants, two additional participants were recruited to replace participants who had correctly predicted the rule.

Stimuli and apparatus The only difference between Experiments 1 and 2 was the presentation of precues. Two Ts with a size of $0.82^{\circ} \times 0.80^{\circ}$ were used as a set of precues to notify participants of the color of the search items before the items were presented. One $\mathrm{T}$, whose center was $1.36^{\circ}$ to the left of the fixation cross, was rotated to the left. The other $\mathrm{T}$, whose center was $1.36^{\circ}$ to the right of the fixation cross, was rotated to the right. The two precues were identical in color, either black or white, and the color always matched the item colors in the search array (see Fig. 3).

Procedure The procedure was identical to that of Experiment 1, except for the addition of precues: After a red fixation cross was shown for $500 \mathrm{~ms}$, white or black precues were presented with the fixation for $500 \mathrm{~ms}$, followed by a $500-\mathrm{ms}$ fixation screen. Participants were instructed that the color of items in a search display would always be identical to the color of the precues. The numbers of trials, blocks, and phases were identical to those in Experiment 1.

\section{Results}

The same criteria as in Experiment 1 were applied in order to exclude participants and trials from the analysis. After removing $1.10 \%$ of the trials with RTs exceeding $10 \mathrm{~s}$, the overall accuracy was $98.90 \%(S D=0.97 \%)$. Only the RTs for correctly answered trials were used in the analysis.

Training phase Probability (valid-rich, invalid-rich, neutralsparse) and block (1-8) were the two main factors of a repeated measures ANOVA. The main effect of block displayed a reduction in RTs, $F(7,245)=12.644, p<.001, \eta^{2}=.265, \mathrm{BF}_{10}=$ $1.946 \mathrm{e}+14$, implying a practice effect (see Fig. 4). The main effect of probability was statistically significant, $F(2,70)=$ 16.676, $p<.001, \eta^{2}=.323, \mathrm{BF}_{10}=2.655 \mathrm{e}+08$. Pairwise contrasts revealed significantly faster RTs in the valid-rich $(M=$ 

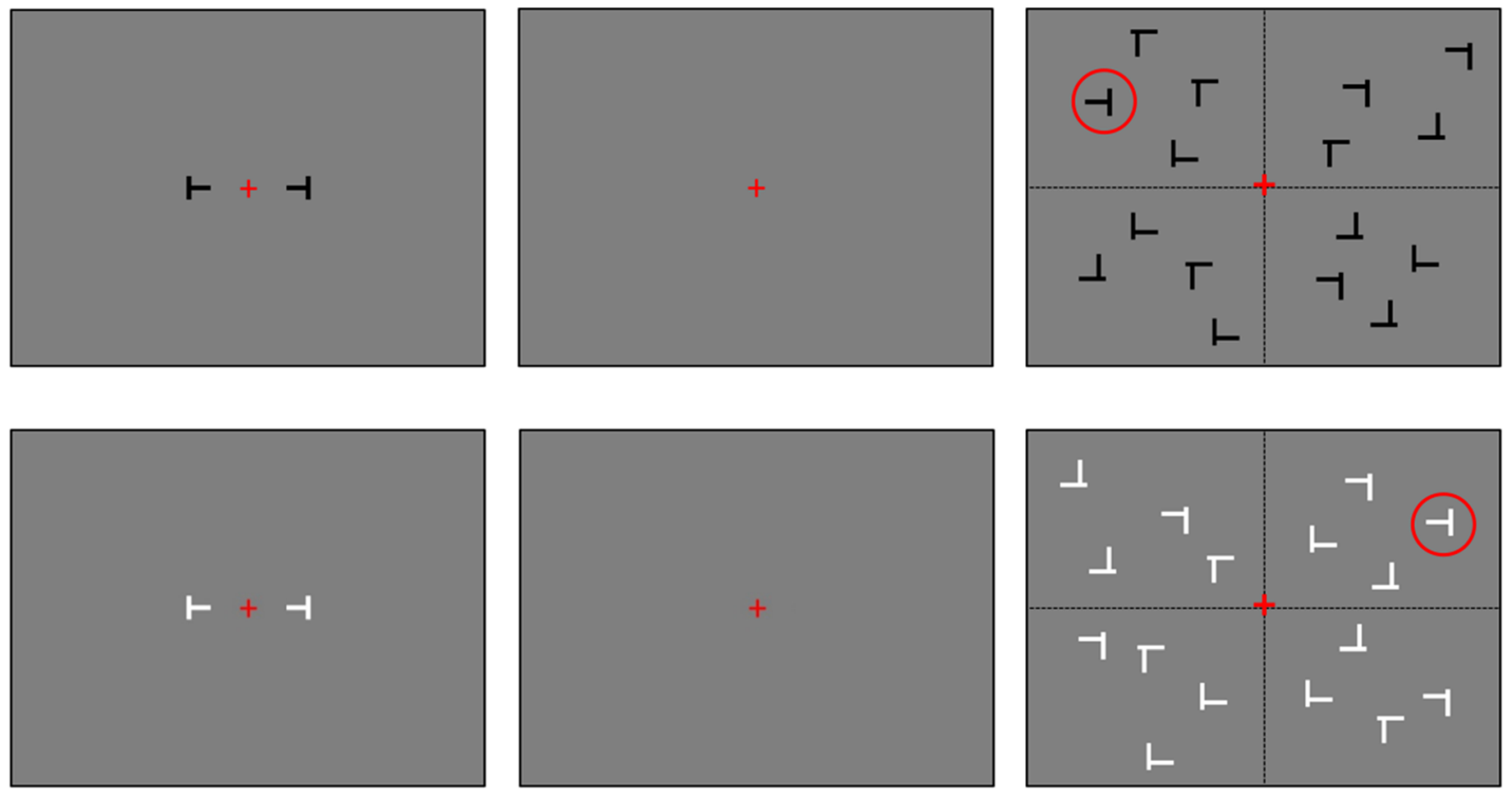

\section{$500 \mathrm{~ms}$}

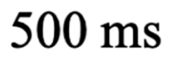

\section{Until Response}

Fig. 3 The procedure of Experiment 2. The colors of the precues and the search items were always identical. Items are not drawn to scale, and both the quadrant borders and the circles on the targets were invisible in the experiment.

$2,712.96 \mathrm{~ms}, S D=390.23 \mathrm{~ms})$ and the invalid-rich $(M=$ $2,810.50 \mathrm{~ms}, S D=491.53 \mathrm{~ms})$ conditions than in the neutralsparse $(M=3,037.17 \mathrm{~ms}, S D=406.71 \mathrm{~ms})$ condition, $F(1,35)=$ $34.020, p<.001, \mathrm{BF}_{10}=1.393 \mathrm{e}+14$, and $F(1,35)=11.237, p=$ $.002, \mathrm{BF}_{10}=3,021.160$, respectively. The difference in RTs between the valid-rich and invalid-rich conditions was also significant, but its evidence anecdotally supported the null hypothesis, $F(1,35)=4.203, p=.048, \mathrm{BF}_{01}=1.170$. The significant context effect seems like a false alarm, given the anecdotal preference for the null hypothesis and the disappearance of the context effect in the testing phase (see the results in the Testing phase and Discussion sections below). The interaction was not significant, $F(8.502,297.586)=1.509, p=.149, \eta^{2}=.041, \mathrm{BF}_{01}=64.258$.

Testing phase In the testing phase, in which the target appeared in the four quadrants with equal probabilities, a 3 (probability: valid-rich, invalid-rich, neutral-sparse) $\times 2$ (block: 9, 10) repeated measures ANOVA was conducted. The main effect of probability was significant, $F(2,70)=9.950, p<.001, \eta^{2}=.221, \mathrm{BF}_{10}$ $=5,651.740$. The RTs of the valid-rich $(M=2,362.14 \mathrm{~ms}, S D=$ (a)

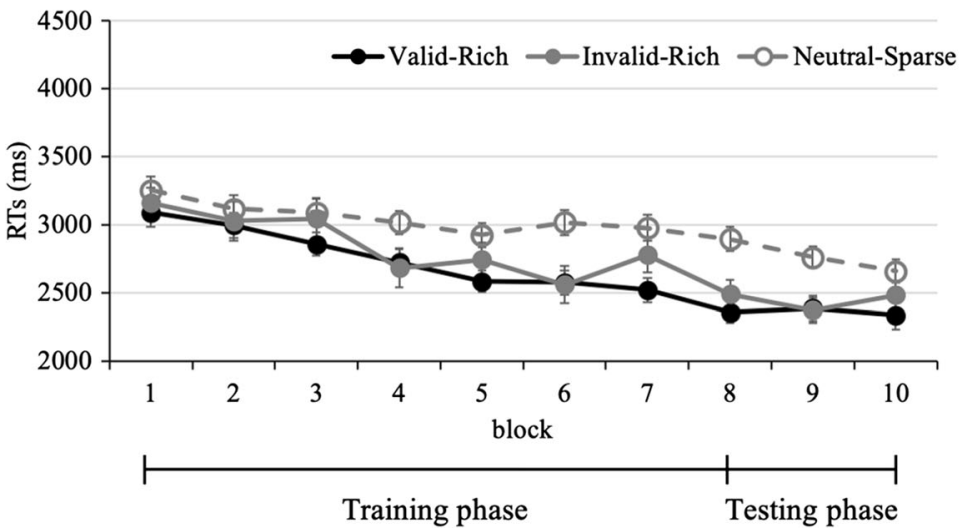

\section{(b)}

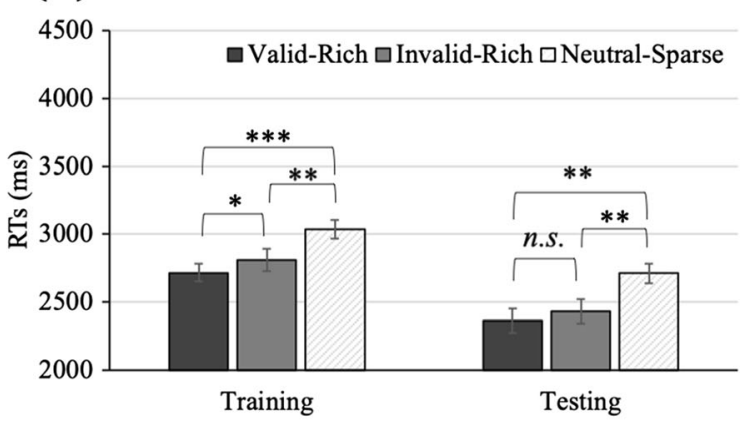

Fig. 4 Results of Experiment 2. (a) Reaction times (RTs) of each probability condition over time. The training phase consisted of Blocks 1-8, and the testing phase consisted of Blocks 9 and 10. (b) RTs by probability and phase. Error bars represent standard errors. ${ }^{*} p<.05,{ }^{* * *} p<.01,{ }^{* * *} p<.001$. 
$390.23 \mathrm{~ms}$ ) condition were significantly faster than the RTs of the neutral-sparse $(M=3,037.17 \mathrm{~ms}, S D=406.71 \mathrm{~ms})$ condition, $F(1,35)=14.056, p=.001, \mathrm{BF}_{10}=1,453.972$, and so were the RTs of the invalid-rich $(M=2,429.97 \mathrm{~ms}, S D=491.53 \mathrm{~ms})$ condition, $F(1,35)=12.125, p=.001, \mathrm{BF}_{10}=110.521$, showing a probability cueing effect. However, the RTs of the valid-rich condition were not significantly faster than the RTs of the invalid-rich condition, $F(1,35)=0.797, p=.378, \mathrm{BF}_{01}=$ 5.017 , implying moderate evidence for a context-independent spatial bias, even when context information was provided before the search array. The main effect of block and the interaction between block and probability were not significant, $F(1,35)=$ $0.082, p=.777, \mathrm{BF}_{01}=6.649, \eta^{2}=.002 ; F(2,70)=1.815, p=$ $.170, \eta^{2}=.049, \mathrm{BF}_{01}=4.320$.

Additional analysis also verified that the context-specific spatial bias was not weakened to become a context-independent spatial bias. When the configuration of the rich quadrants (adjacent or diagonal) was included as another factor in the repeated measures ANOVA, the three-way interaction was not significant, $F(2,68)=0.118, p=.889, \eta^{2}=.000, \mathrm{BF}_{01}=5.38$. The two-way interaction of probability and configuration was significant, $F(2$, $68)=3.207, p=.047, \eta^{2}=.021, \mathrm{BF}_{10}=4.148$. However, there was no difference in the trend of the context-related spatial bias, in that both the adjacent group and the diagonal group showed context-independent spatial biases: adjacent, $t(68)=-0.571, p=$ $.570, \mathrm{BF}_{01}=4.97$; diagonal, $t(68)=-0.609, p=.545, \mathrm{BF}_{01}=$ 3.96. In sum, a context-independent spatial bias was consistently observed, regardless of the configuration of the search quadrants.

Through an additional analysis including hemifield (left, right) as a between-subjects factor, we tested the possible interference of a leftward search habit with the context-independent spatial bias. We found no evidence of a leftward search bias overall, $F(1,10)=2.40, p=.152, \eta^{2}=.094, \mathrm{BF}_{01}=1.132$, or of its influence on the spatial bias, $F(2,20)=0.759, p=.481, \eta^{2}=$ $.015, \mathrm{BF}_{01}=3.300$.

\section{Discussion}

Experiment 2 was conducted to rule out the possibility that a context-independent spatial bias was induced by lack of time to process the context information. We provided precues $1,000 \mathrm{~ms}$ before the search began, but still we found no evidence of a context-specific spatial bias. The failure of context-specific probability learning in Experiment 2 was not likely due to a lack of time for top-down attention to function or for the formation of a context template, because it has been shown that $1 \mathrm{~s}$ is sufficient for attentional control to be exploited (Han \& Kim, 2009).

It can be argued that top-down attention would not be used in context-related statistical learning, resulting in a contextindependent spatial bias. We could not directly check whether the participants attended to the precues, due to the lack of a secondary task (e.g., pressing the "W" vs. "B" button at the beginning of the trial), and thus could not ensure that participants processed the context information. However, this possibility is made less feasible, considering the decreased RTs in Experiment $2(M=2,779.64 \mathrm{~ms}, S D=361.45 \mathrm{~ms})$ than in Experiment $1(M=$ $3,007.58 \mathrm{~ms}, S D=452.07 \mathrm{~ms}), t(70)=2.363, p=.021, \mathrm{BF}_{10}=$ 2.549 , for the total trials; $t(70)=2.300, p=.024, \mathrm{BF}_{10}=2.259$, for the training phase; $t(70)=2.304, p=.024, \mathrm{BF}_{10}=2.278$, for the testing phase. The faster RTs may have been due to maintenance of the target color in working memory (Han \& Kim, 2009; Soto, Heinke, Humphreys, \& Blanco, 2005) or to a priming effect from the target color- and shape-identical precues. The exact reason for this difference is not known, but the faster RTs suggest the possibility that the precues were attended in order to facilitate the visual search.

Given that the precues were attended to, maintaining the precues in working memory could have yielded an interference effect on location probability learning, due to the working memory load. Though maintaining a search template does require working memory, the load imposed by maintaining a single attentional template over time should be minimal (Carlisle, Arita, Pardo, \& Woodman, 2011). Furthermore, though a secondary task (e.g., spatial working memory) could impair the expression of learned statistical knowledge in a contextual cueing paradigm (Annac et al., 2013; Annac, Zang, Müller, \& Geyer, 2019), probability cueing has been shown to be immune to interference from spatial working memory load, unlike contextual cueing (Won \& Jiang, 2015).

Though the RTs differed significantly between the valid-rich and invalid-rich conditions in the training phase, the effect completely disappeared in the testing phase. Therefore, it would be unconvincing to define the result in the training phase as a context effect. The reason why we observed a contextindependent spatial bias instead of a context-specific attentional bias may lie in the lack of relevance of the context to the task. In Experiments 1 and 2, the color provided beneficial information about which quadrant the target would frequently appear in. However, it was still possible to perform the task without the color information, because there was only one target in an array. Because the item color was not very informative, the precues might not have been fully processed in order to make a distinctive context template. If context were highly relevant to the task, such that it was impossible to perform the task without knowing the context, then participants might process the context information and use the context-specific probabilistic knowledge.

\section{Experiment 3}

In Experiment 3, we examined whether task relevance can promote the learning of a context-specific spatial bias. To increase task relevance, we modified the experiment in such a way that participants could not perform the visual search task unless they processed the color information before the search began. In Experiment 3, both a black and a white T were presented among 
black and white Ls in a search display, and participants were precued as to the color of the target they were to search for. Thus, participants were not able to maintain a high accuracy during the search task unless they maintained the context information. We now expected to find a context-specific spatial bias, as the context needed to be fully processed in order to perform the search task.

\section{Method}

Participants Thirty-nine new participants took part in Experiment 3 for a monetary reward. The exclusion criteria for participants were identical to those in Experiments 1 and 2, resulting in 36 sets of data.

Stimuli and apparatus Two Ts and $30 \mathrm{Ls}$ were presented in a search array; one of the Ts and 15 of the Ls were white, and the remaining items were black. The target in the given context was pointing either to the left or the right with equal probabilities. The $\mathrm{T}$ for the opposite context was also pointing either to the left or the right, but the direction was randomly selected, independent of the target orientation. The other apparatus and stimuli were identical to those of Experiments 1 and 2.
Procedure The numbers of practice trials, blocks, and experimental trials were identical to those in Experiments 1 and 2. A schematic display of a trial is presented in Fig. 5.

After a 500-ms fixation display, a predefined set of precues, in white or black, appeared for $500 \mathrm{~ms}$ and then disappeared for $500 \mathrm{~ms}$. A search display containing 32 items was then given until response. The task was to find the target with the same color as the precues and to report the side to which the target was rotated. The probability of the black or the white $\mathrm{T}$ appearing in each rich quadrant was manipulated, regardless of whether or not the color was cued. As a result, the probabilistic manipulation throughout the experiment was kept the same as in Experiments 1 and 2.

An awareness check immediately followed the experiment, in which the participants were asked the same questions as in Experiments 1 and 2.

\section{Results}

Trials on which RTs exceeded $10 \mathrm{~s}$ were removed, and RTs only for correct responses were used in the analysis. In all, $2.96 \%$ of the total trials were removed, and the mean accuracy was $97.13 \%$ $(S D=4.08 \%)$. The results by blocks and by phase are plotted in Fig. 6.
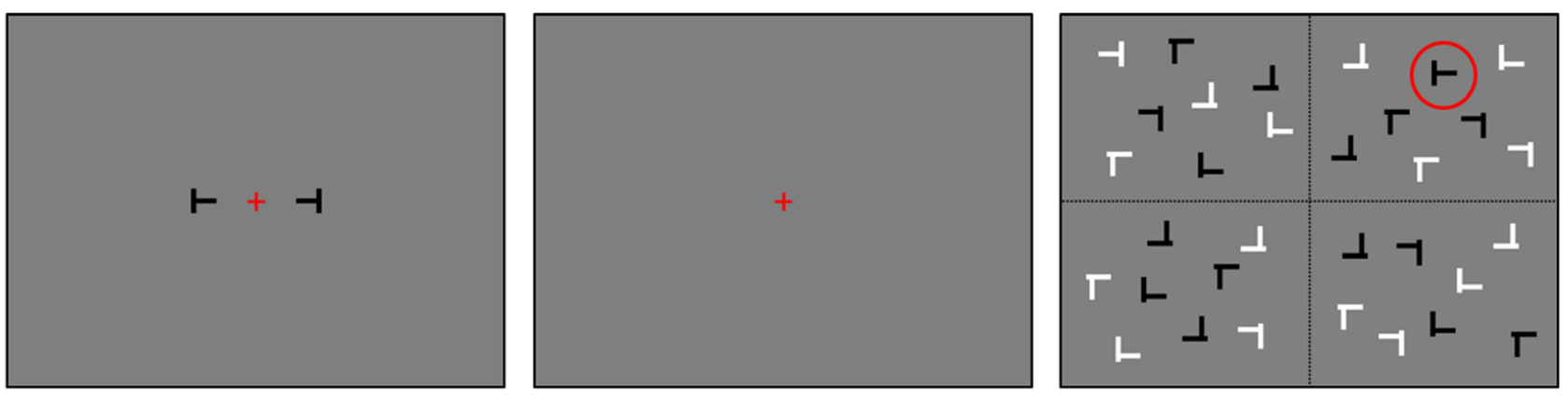

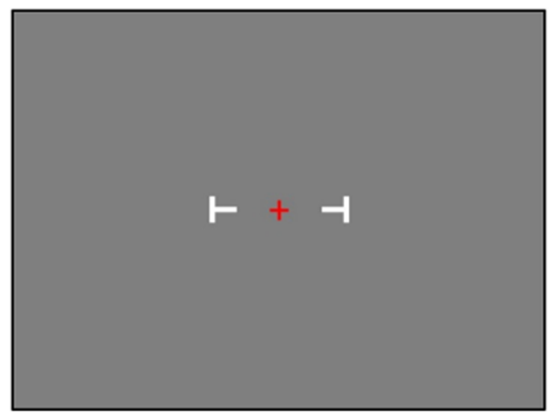

$500 \mathrm{~ms}$

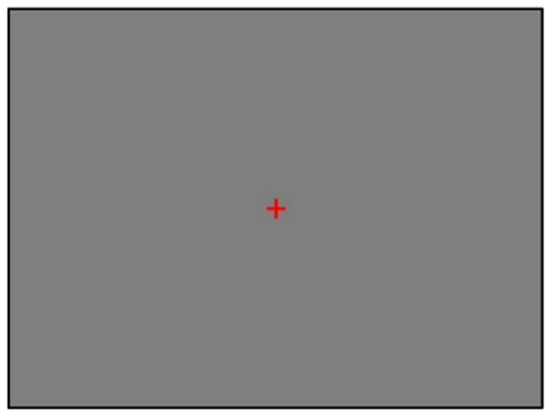

$500 \mathrm{~ms}$

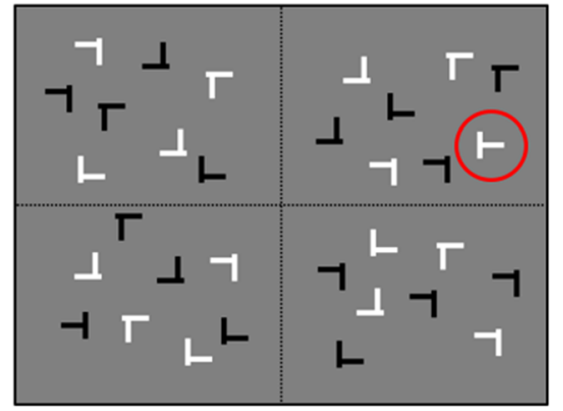

Fig. 5 The procedure of Experiment 3. The target color was given by the precues. The upper row indicates a trial with a black context, and the bottom row indicates a trial with a white context. The items are not drawn to scale, and the quadrant borders and the circles on the target were not shown in the experiment. 
(a)

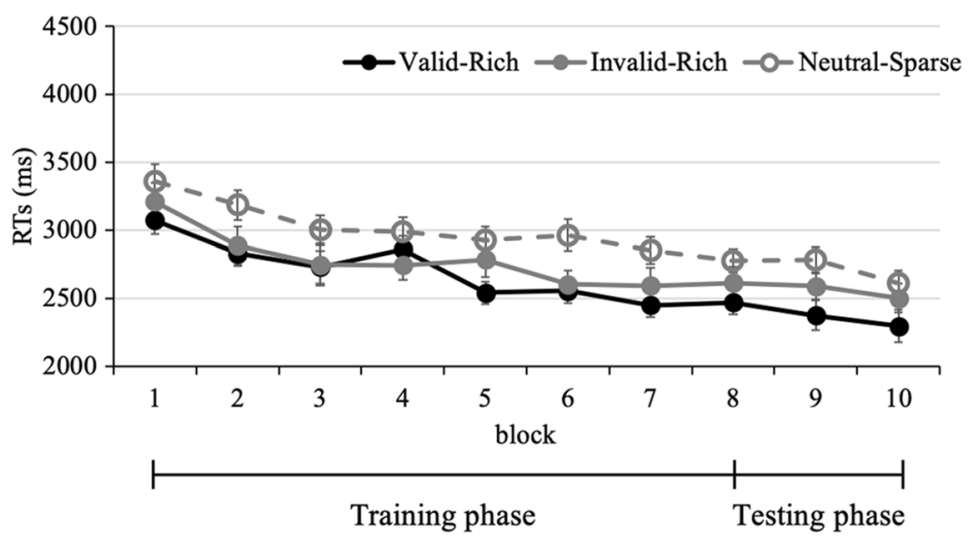

(b)

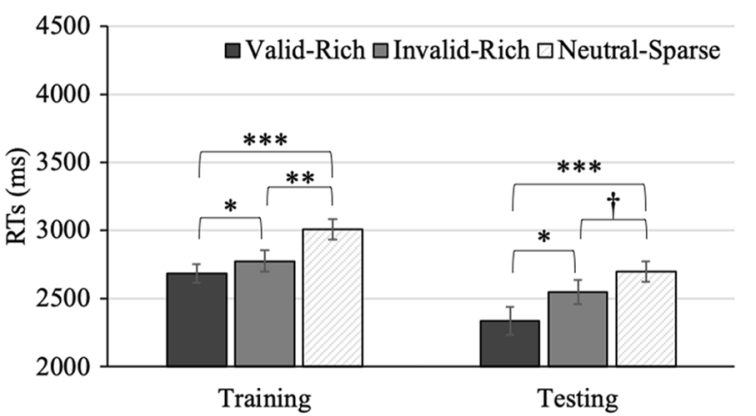

Fig. 6 Results of Experiment 3. (a) Reaction times (RTs) by blocks and (b) results grouped by probability and phase. Error bars represent standard errors. ${ }^{\dagger} p<.1,{ }^{*} p<.05,{ }^{* *} p<.01,{ }^{* * *} p<.001$

Training phase A 3 (probability: valid-rich, invalid-rich, neutral-sparse) $\times 8$ (block: 1-8) repeated measures ANOVA was conducted. The main effect of block was significant, $F(7,245)$ $=11.574, p<.001, \eta^{2}=.249, \mathrm{BF}_{10}=1.273 \mathrm{e}+13$, suggesting a practice effect. The main effect of probability was also significant, $F(1.404,49.127)=14.893, p<.001, \eta^{2}=.298, \mathrm{BF}_{10}=$ 6.290e+07. Pairwise contrasts yielded a significant probability cueing effect: The RTs of the valid-rich $(M=$ $2,688.34 \mathrm{~ms}, S D=408.64 \mathrm{~ms})$ and the invalid-rich $(M=$ 2,779.07 $\mathrm{ms}, S D=456.14 \mathrm{~ms}$ ) conditions were significantly faster than the RTs of the neutral-sparse $(M=3,007.57 \mathrm{~ms}, S D$ $=462.32 \mathrm{~ms}$ ) condition, $F(1,35)=24.756, p<.001, \mathrm{BF}_{10}=$ $5.577 \mathrm{e}+10$, and $F(1,35)=10.016, p=.003, \mathrm{BF}_{10}=6,284.396$, respectively. A context effect was also observed, even though this effect was not supported by the Bayesian analysis, $F(1,35)$ $=5.091, p=.030, \mathrm{BF}_{01}=2.094$. The interaction between probability and block was not significant, $F(14,490)=0.652, p=$ $.821, \eta^{2}=.018, \mathrm{BF}_{01}=1,183.238$.

Testing phase The two main factors were probability (validrich, invalid-rich, neutral-sparse) and block $(9,10)$. A repeated measures ANOVA revealed that the main effect of block and the interaction between probability and block were not significant, $F(1,35)=3.100, p=.087, \eta^{2}=.081, \mathrm{BF}_{01}=1.702$, and $F(1,35)=0.335, p=.716, \eta^{2}=.009, \mathrm{BF}_{01}=14.086$. The main effect of probability was significant, $F(2,70)=8.923, p<.001$, $\eta^{2}=.203, \mathrm{BF}_{10}=823.368$. A probability cueing effect was observed between the valid-rich $(M=2,338.77 \mathrm{~ms}, S D=$ $616.29 \mathrm{~ms})$ and the neutral-sparse $(M=2,699.62 \mathrm{~ms}, S D=$ $467.10 \mathrm{~ms})$ conditions, $F(1,35)=16.547, p<.001, \mathrm{BF}_{10}=$ 889.287. The RTs between the invalid-rich $(M=2,548.13 \mathrm{~ms}$, $S D=530.13 \mathrm{~ms}$ ) and the neutral-sparse conditions was not statistically significant, $F(1,35)=3.263, p=.079, \mathrm{BF}_{01}=$ 1.053. Importantly, when the context became relevant to a task, a context effect was shown in which the RTs of the valid-rich condition were significantly faster than those of the invalid-rich condition, $F(1,35)=6.160, p=.018, \mathrm{BF}_{10}=6.563$. The results moderately support a context-specific spatial bias: Probabilistic knowledge was distinctively formed by different contexts, and the context information was used to predict the frequent target location.

\section{Experiment 4}

To generalize the results of Experiment 3 and reject the possibility that the increase in the set size of Experiment 3 could have influenced the spatial bias, we conducted Experiment 4. In Experiment 4, participants performed a visual search task with another type of search stimulus: The search items were black or white squares with gaps along the edges. A single display contained either black or white items, as in Experiments 1 and 2 , but the location of the relevant gap (left vs. right or top vs. bottom) changed, depending on the color of the items. The operational definition of context was the combination of item colors (black or white), the target location (horizontal or vertical), and the response dimension (left or right hand).

\section{Method}

Participants A total of 37 participants participated for a monetary reward. In all, 36 sets of data remained after the exclusion of one dataset, using the same criteria as in the other experiments.

Stimuli and apparatus The precue was either a black- or a white-filled square of $1.6^{\circ} \times 1.6^{\circ}$ in size, presented at the center of the display. Sixteen square frames with small gaps in the middle of every side were presented as the search stimuli. Each stimulus was $0.82^{\circ} \times 0.80^{\circ}$ of visual angle in size, and the gaps were $0.16^{\circ}$ wide. Among the 16 stimuli, one stimulus was designated as the target, with two larger gaps, of length $0.27^{\circ}$, each positioned on the horizontal and vertical axis. All 16 search items in a single search array had the same color, either black or white. 
The other apparatus and the stimuli that were not mentioned above were identical to those in Experiments 1, 2, and 3.

Procedure The procedures and the trial sequence were identical to those of Experiment 3, except in the respects mentioned below. The search display contained 16 items, and the task was to report the location of a targeted larger gap. The response dimension was defined according to the color context: If the search items were white, participants used their left hand to press either the " $z$ " or the " $x$ " key, to report the larger gap on the left or the right side of the square. If the search items were black, participants used their right hand to press either the "." or the " " key, to report the larger gap on either the top or the bottom side of the square. The color and the response dimension were counterbalanced across participants. A schematic display of Experiment 4 is depicted in Fig. 7.

\section{Results}

The analysis criteria from Experiment 3 were again applied, removing the $5.03 \%$ of total trials whose RTs exceeded $10 \mathrm{~s}$. The accuracy was $99.24 \%$ on average $(S D=0.76 \%)$. The RTs by blocks and by phases are shown in Fig. 8 .

Training phase A 3 (probability: valid-rich, invalid-rich, neutral-sparse $) \times 8$ (block: $1-8)$ repeated measures ANOVA yielded a significant spatial bias, $F(1.619,56.679)=7.429, p=.003, \eta^{2}=$ $.175, \mathrm{BF}_{10}=1,072.935$, and a significant practice effect, $F(7$, $245)=34.614, p<.001, \eta^{2}=.497, \mathrm{BF}_{10}=1456 \mathrm{e}+41$. Pairwise comparisons revealed that the difference between the RTs of the valid-rich $(M=3,281.71 \mathrm{~ms}, S D=650.15 \mathrm{~ms})$ and the invalidrich $(M=3,389.24 \mathrm{~ms}, S D=720.20 \mathrm{~ms})$ conditions was not statistically significant, $F(1,35)=3.314, p=.077, \mathrm{BF}_{01}=$ 1.601 , but a numerical trend existed for a spatial bias toward the context-predicted rich quadrant. The difference between the RTs of the invalid-rich and neutral-sparse $(M=3,569.72 \mathrm{~ms}, S D$ $=619.83 \mathrm{~ms}$ ) conditions was also not significant, $F(1,35)=$ $3.787, p=.060, \mathrm{BF}_{10}=6.652$. The RTs of the valid-rich condition were faster than those of neutral-sparse condition, $F(1,35)=$ 16.442, $p<.001, \mathrm{BF}_{10}=5.536 \mathrm{e}+7$. The interaction between probability and block was not significant, $F(8.623,301.792)=$ $0.721, p=.683, \eta^{2}=.020, \mathrm{BF}_{01}=922.211$.

Testing phase Probability (valid-rich, invalid-rich, neutral sparse) and block (9 and 10) were included as two factors for the repeated measures ANOVA. Neither the main effect of block nor the interaction between probability and block was statistically significant, $F(1,35)=2.293, p=.139, \eta^{2}=.061, \mathrm{BF}_{10}=2.198$, and $F(2,70)=0.470, p=.627, \eta^{2}=.013, \mathrm{BF}_{01}=8.635$. The main effect of the probability was significant, $F(2,70)=6.747, p$ $=.002, \eta^{2}=.162, \mathrm{BF}_{10}=23.888$. Pairwise comparisons revealed that the RTs of the valid-rich $(M=2,697.91 \mathrm{~ms}, S D=617.23$
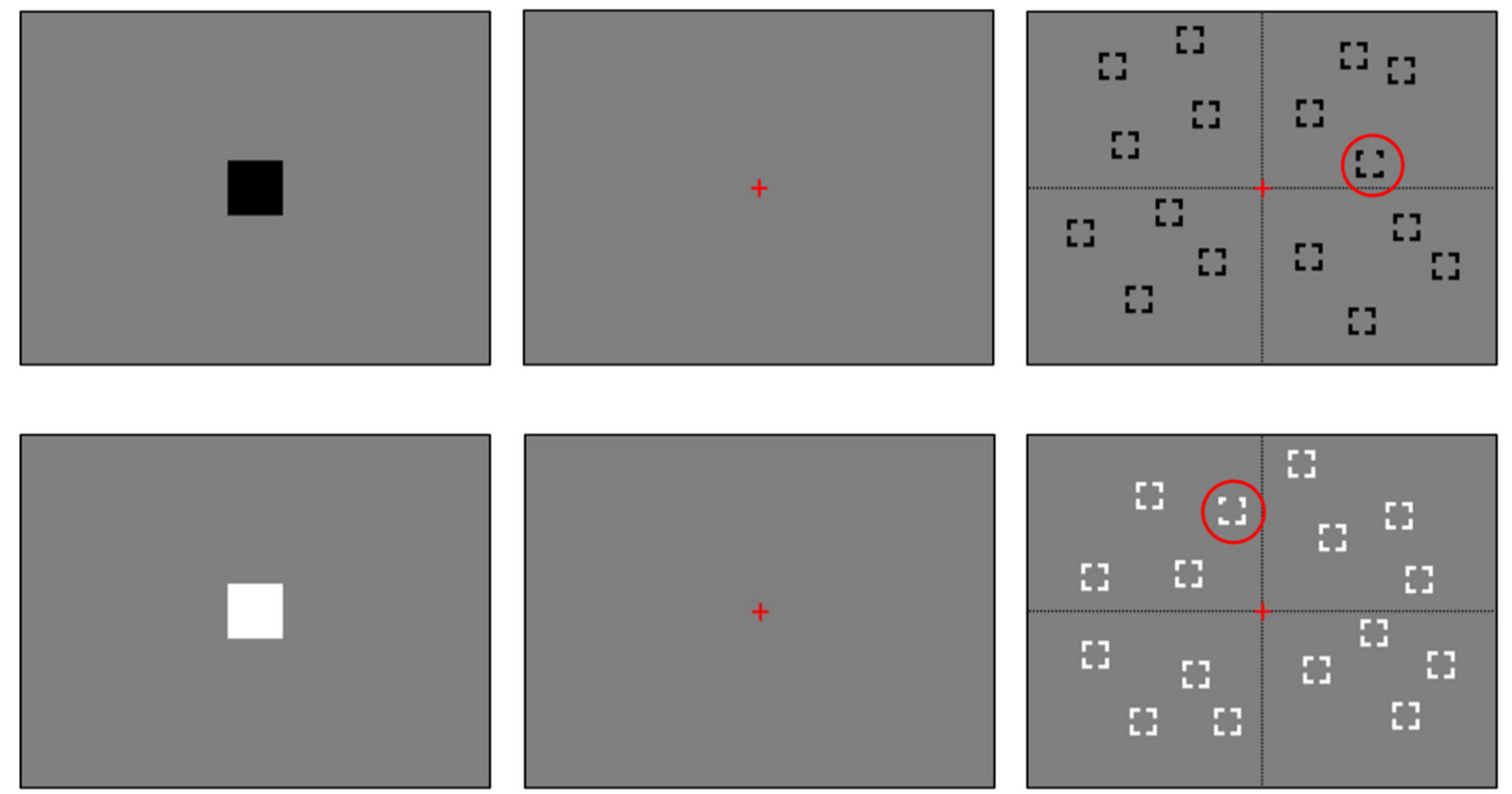

\section{$500 \mathrm{~ms}$}

$500 \mathrm{~ms}$

\section{Until Response}

Fig. 7 The procedure of Experiment 4. The colors of the precues and the search items were always the same. The items are not drawn to scale, and the quadrant borders were invisible in the experiment. The circles were also invisible to participants. 
(a)

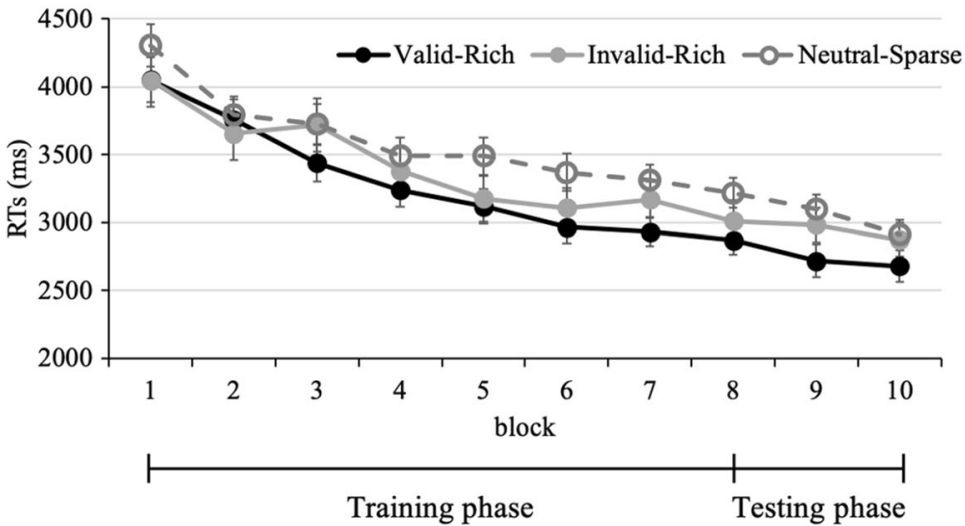

(b)

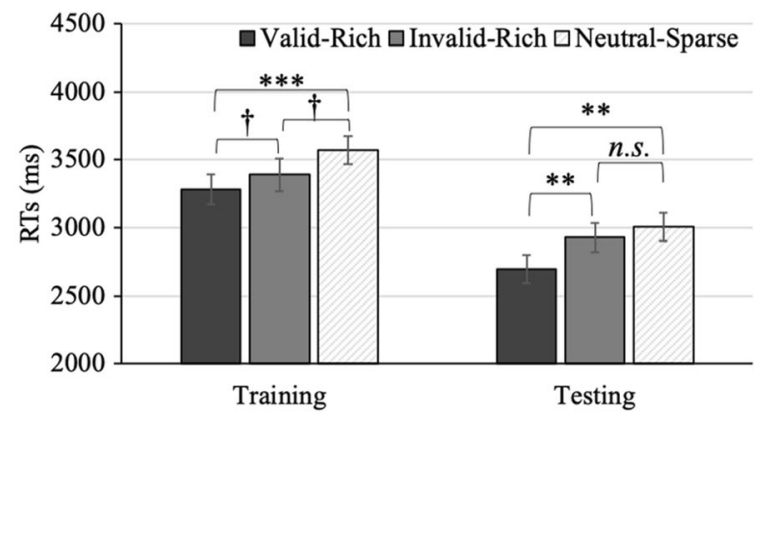

Fig. 8 Results of Experiment 4. (a) Reaction times (RTs) by blocks. (b) Results grouped by probability and phase. Error bars represent standard errors. ${ }^{\dagger} p$ $<.1,{ }^{* * *} p<.01, \stackrel{* * * *}{p} p<.001$.

$\mathrm{ms})$ and the neutral-sparse $(M=3,004.33 \mathrm{~ms}, S D=625.35 \mathrm{~ms})$ conditions were significantly different, $F(1,35)=12.743, p=$ $.001, \mathrm{BF}_{10}=93.423$, and that the RTs of the invalid-rich $(M=$ $2,928.90 \mathrm{~ms}, S D=641.06 \mathrm{~ms}$ ) and neutral-sparse conditions were not different, $F(1,35)=0.616, p=.438, \mathrm{BF}_{01}=5.341$. Importantly, the RTs of the valid-rich condition were significantly faster than those of the invalid-rich condition, $F(1,35)=$ 9.240, $p=.004, \mathrm{BF}_{10}=5.647$, replicating the context-specific spatial bias observed in Experiment 3.

\section{Discussion}

Experiments 3 and 4 were designed to assign a high degree of task relevance to the context, so that the context information had to be maintained and utilized in order to select one target (Exp. 3) or one response dimension (Exp. 4). As a result, participants developed context-specific statistical knowledge and guided their spatial attention toward the predicted target location based on context.

The only difference in the designs of Experiments 2 and 3 was whether or not the context information was essential to the task performance. While keeping the set size and the display environment (a single context presented in a search display) the same, we increased the task relevance of the context. Therefore, we rejected the possibility that the context-independent spatial bias of Experiments 1 and 2 had been driven by the display environment. Instead, we verified that task relevance modulated the context-related spatial bias.

There is a possibility that the increase in the set size of Experiment 3 as compared to Experiment 2 might have modulated the context-related spatial bias. Though in Experiment 3 the set size might have affected the degree of the probability cueing effect (Geng \& Behrmann, 2005; Jiang, Swallow, \& Rosenbaum, 2013b; Jiang et al., 2015), the presence of context-specific probability cueing solely due to increased set size is unlikely, as Experiment 4 also revealed a context-specific spatial bias with a set size as identical to that in Experiment 2. Differences in item shape (as compared to Exp. 3) and task difficulty (as compared to Exp. 2) are also unlikely to have affected the task-related spatial bias, because the probability cueing effect can be transferred to different task difficulties and to different search items (Jiang et al., 2015). It seems that implicit spatial bias, rather its contextindependent or context-specific form, is independent of the stimulus properties and reflects the search habit toward the frequent target location. Therefore, the results from Experiments 3 and 4 suggest that implicit learning of context information depends on the situation.

\section{General discussion}

The aim of this study was to find out whether multiple contexts can be learned implicitly and can be used to guide spatial attention in a context-specific manner. The probability of the frequent target location was manipulated, so that different contexts predicted different locations having high target probability. In Experiment 1, a context-independent spatial bias was observed when the context was given simultaneously with the search array. In Experiment 2, participants were given enough time to process the context information before the search array, but a contextspecific spatial bias still did not emerge. However, when the context information became task-relevant in Experiments 3 and 4, a context-specific spatial bias emerged. The contextindependent or context-specific form of the spatial bias was acquired in an incidental and implicit manner, because participants were not explicitly aware of the probability manipulation until the end of the experiments (since those with explicit awareness were excluded from analysis), and because participants had no intention to learn the rule. In sum, implicit learning of a contextrelated spatial bias can be modulated by task relevance. 
Task difficulty sometimes enhances behavioral performance in selective attention (Lavie, 1995, 2005) or perceptual learning tasks (Wang, Song, Qu, \& Ding, 2010). Thus, one might argue that the increase in the set size in Experiment 3 made the task more difficult, and that the increased task difficulty induced a context-specific spatial bias. Nevertheless, we argue that task difficulty is not likely to be a key factor that elicited the contextspecific spatial bias, for the following reasons. First, increased task difficulty does not always enhance learning or task performance. For instance, previous studies have shown that task difficulty does not modulate (Jiang \& Chun, 2001), or sometimes impairs (De Houwer \& Beckers, 2003), task performance. Second, though the set size in Experiment 3 was greater than that in Experiment 2, across all conditions, the behavioral performance between the two experiments did not differ (all $t \mathrm{~s}$ $<1, p s>.05$ ), demonstrating that the set size did not significantly impact search difficulty. Third, context-specific probability learning was revealed in Experiment 4, in which the set size was identical to that in Experiment 2 and task relevance was as high as in Experiment 3. Finally, we conducted an additional experiment to verify that search difficulty did not yield a context-specific spatial bias. We made a difficult version of Experiment 2 by changing the offset of the Ls to make them more similar to the target Ts (Jiang \& Chun, 2001), and tested this display on a separate group of participants. The results showed that task difficulty was successfully manipulated, as the overall RTs increased by $802.08 \mathrm{~ms}$ (equivalent to an effective increase of the set size by 4.62 items). However, a contextspecific spatial bias was still not found with this increased search difficulty. Thus, task relevance, not task difficulty, was a key factor that modulated the context-specific spatial bias in the present study.

When the task was relevant to the search task, visual features were used to segregate each incident into separate sets of statistical knowledge. Previous studies have shown that long-term knowledge about the visual context (visual scene) can drive spatial attention to the target location (Summerfield, Lepsien, Gitelman, Mesulam, \& Nobre, 2006). Summerfield et al. showed that the context can serve as an endogenous cue that facilitates target detection.

However, the context in the present study could not be aligned with the endogenous cue, because the endogenous cue was reported as not interacting with the probability cueing. For example, when both the explicit context (background visual scene) and the implicit context (distractor configuration) predicted the target locations, explicit context, and not implicit context, guided spatial attention to the target location, preventing the impact of implicit context (Rosenbaum \& Jiang, 2013). Also, when an endogenous arrow cue was presented with the probability cue, learning of the probability cue failed (Jiang, Swallow, \& Rosenbaum, 2013b). Unlike in these previous studies, the context in the present study served as an informative cue coexisting with the location probability cue.
Given that an endogenous cue does not interact with location probability learning, the context in this study would not be included under the scope of an endogenous cue. Though the context of this research functioned as an endogenous cue, it shared more common aspects with a habit: It was gradually and implicitly acquired through experience (Jiang, 2018; Jiang \& Sisk, 2019; Jiang, Swallow, \& Sun, 2014a).

The definition of context has varied across the topics that each study has dealt with. In the classical study on contextual cueing by Chun and Jiang (1998), the context was the spatial configuration of the distractors that were associated with the target location. When a configuration of distractors was shown repeatedly, the search times for the target decreased, meaning that the context was used to guide spatial attention to the target location. In another contextual cueing study, the color of the items was used as the context (Jiang \& Song, 2005). Half of the repeatedly presented configurations were displayed when the search items were presented in black, and the other half of the repeated configurations were presented when the search items were presented in white. When the participants were trained with both black and white contexts, they were able to form a color-specific memory of the configurations. That is, the color served as a context that predicted which configuration to use for target location guidance. In other studies, the location of items was also defined as a context that predicted the proportion of congruency in a Stroop task, an Eriksen flanker task, and Simon tasks (Crump et al., 2006; Hübner \& Mishra, 2016; Wendt et al., 2008). In the present study, we defined context as either the color of the items or the response dimension, and showed that both types of context can be used as a task-informative cue. The present results, taken together with those of previous studies, suggest that the visual system can extract information from a wide range of visual inputs to increase task performance.

In Jiang and Song (2005), participants were able to form a color-specific memory for repeated configurations when the search items were presented in either black or white during contextual cueing experiments. In their study, only one target item was presented in the search display, and thus the color of the items was not critical for the search. In contrast, the participants in the present study failed to acquire context-specific knowledge in Experiments 1 and 2 when the color of the items were not required for the search. This discrepancy could be explained by differences between the experimental paradigms. Though both contextual cueing and probability cueing are based on incidental learning (Jiang, Swallow, \& Capistrano, 2013a; Jiang \& Sisk, 2019; Jiang, Swallow, \& Rosenbaum, 2013b; Jiang, Swallow, \& Sun, 2014a), what is learned in these two paradigms is not exactly the same. The contextual cueing effect occurs because of the individual memory trace of the association between the target location and the distractor configurations (Chun, 2000; Chun \& Jiang, 1999). In Jiang and Song's study, it was possible that each association between the distractor configuration in a specific color and a target location could be 
learned as an independent representation. In contrast, in the probability cueing paradigm, what participants should have learned is not an association between an individual target location and the context, but the overall probability distribution computed across multiple trials in a given context. To accumulate statistical information from each trial separately for each context, active processing of the context information was required. Thus, unlike for Jiang and Song, the task relevance of the context information was a prerequisite for context-specific learning in the present study.

Several factors can be considered to have resulted in the context-independent spatial bias in Experiments 1 and 2, even though a context-specific spatial bias was possible. The first possibility is the reduced overall rich-to-sparse ratio, as compared to other studies of the probability cueing effect. The size of the cueing effect has a linear relationship to the probability difference (Ferrante et al., 2018; Schwark \& Dolgov, 2013). We expect that the cueing effect in our study must have been handicapped, because the overall probability of target presence in each rich quadrant was $33 \%$, which was smaller than the $50 \%$ probability of a typical probability cueing effect paradigm. However, though the cueing effect was weakened due to a reduced rich-to-sparse ratio, it is unlikely that the reduced cueing effect made the spatial bias insensitive to context information. Although the probability cueing effect was driven by RTs in the neutral-sparse condition minus RTs in the valid-rich and invalid-rich conditions, a measure that was sensitive to the reduced rich-to-sparse ratio, the context effect was driven by the RT difference of valid-rich and invalid-rich conditions, which was not sensitive to rich-tosparse ratio. The probabilities of target appearance were identical across the two rich quadrants and across four experiments. The context effect was also numerically greater when task relevance was high (209 ms in Exp. 3 and $231 \mathrm{~ms}$ in Exp. 4 vs. $4 \mathrm{~ms}$ in Exp. 1 and $68 \mathrm{~ms}$ in Exp. 2). Therefore, it is unlikely that the small probability ratio caused insensitivity to a context-specific spatial bias.

Another possible cause of the context-independent spatial biases is weaker reinforcement of attentional shift due to the intermixed contexts. The probability cueing effect occurs because of a vector that is an outcome of the reinforcement of attentional shift toward a location where the target is successfully detected (Jiang, 2018; Jiang, Swallow, \& Capistrano, 2013a). Two contexts were intermixed in this research, so that the vectors of a context should have been interdependent with the vectors of another context. However, intermixed contexts are also unlikely to have caused a context-independent spatial bias, because Experiments 3 and 4 also had randomly intermixed contexts.

The third possible cause of the context-independent spatial biases is that the context-specific probability experience failed to be consolidated as long-term statistical knowledge. Although it disappeared in the testing phase, a context-specific spatial bias was weakly shown in the training phases of Experiments 1 and 2. However, Bayes factors implied that the context-specific spatial bias was not very convincing. Bayes factors, which provided quantified evidence for either the alternative model (contextspecific spatial bias) or the null model (context-independent spatial bias), rather moderately or anecdotally supported the contextindependent spatial bias in the training phases of Experiments 1 and 2. Also, the length of the training phase was enough to allow consolidation, because the probability cueing effect is acquired rapidly (Jiang, Swallow, Rosenbaum, \& Herzig, 2013c): According to Salovich, Remington, and Jiang (2018), 24 trials of a rich condition enable covert attention to be biased (with another 24 trials being required for a sparse condition), and 48 trials of a rich condition enable overt attention to be biased (with another 48 trials for a sparse condition). We provided 96 trials of rich condition per each context, and this number exceeds the minimum number of trials for learning.

The failure to observe a context-specific spatial bias in Experiments 1 and 2 is consistent with the findings of Won and Jiang (2015). In their study, a visual search task in the probability cueing paradigm was performed with and without a spatial working memory load. Here, the load and no-load conditions predicted different locations as the rich locations, but this context information was not critical for accurate visual search. Their results showed that participants failed to use the spatial working memory contents to enhance visual search performance. Though spatial working memory and visual search share common cognitive mechanisms (Oh \& Kim, 2004; Woodman \& Luck, 2004), this was not sufficient to induce a context-specific spatial bias. These results suggest that context information should be actively processed during visual search to enable contextspecific probability learning.

It might be more cognitively demanding to learn two sets of probability distributions separately according to contexts than to learn a single set (i.e., the average probability distribution of both contexts). Indeed, neural activation shows a pattern of an annulment of benefits or bottleneck of a process when dual regularities exist (Davis \& Hasson, 2018). Though behavioral performance can be maximized by learning two sets of probability distributions, computational load increases as well. The visual system would thus learn context information when the benefit is greater than the cost, and task relevance can be one of the key factors that contributes to the benefit. When task relevance is low (Exps. 1 and 2), visual search can be conducted without context information, and thus the cost of learning that context information exceeds the benefit (e.g., faster search), resulting in the failure of context-specific learning. However, it was impossible to perform the search task without context information in Experiments 3 and 4, and the benefit to visual search exceeded the cost of learning the context information. Accordingly, the learning of context-specific information is likely to be an outcome of a strategy to maximize the net benefit and minimize the possible cost.

Recent research has proposed habitual attention as a new type of attentional resource (Jiang, 2018; Jiang, Koutstaal, \& Twedell, 
2016; Jiang et al., 2018; Jiang, Swallow, \& Capistrano, 2013a; Jiang, Swallow, \& Rosenbaum, 2013b; Jiang, Won, \& Swallow, $2014 b$ ), one that is not easily explained by the top-down/bottomup attention dichotomy (Awh, Belopolsky, \& Theeuwes, 2012). Habitual attention is distinguished from explicit, goal-driven attention in that incidental learning through habitual attention emerges gradually, in an unconscious manner, and is inflexible, such that statistical knowledge is insensitive to outcome devaluation (Jiang \& Sisk, 2019). Cognitive processes that involve habitual attention also are not influenced by working memory load (Annac et al., 2013; Annac et al., 2019; Vickery, Sussman, \& Jiang, 2010; Won \& Jiang, 2015) or endogenous cues (Jiang, Swallow, \& Rosenbaum, 2013b). Habitual attention is an attentional resource that is conceptually and functionally dissociable from other types of attentional resources.

Location probability learning is one of the cognitive processes that uses habitual attention in its operation. An implicit memory of the probability distribution is gradually built up by the repetitive shifting of spatial attention. In a reverse process, spatial attention is shifted on the basis of the implicit memory according to the probability distribution. Such a bidirectional relation between implicit memory and spatial attention results in location probability learning and the use of habitual attention.

The present study further suggests that habitual attention can flexibly be adjusted, depending on the task relevance of the context information. To form context-specific knowledge, two sets of knowledge should be accumulated in parallel. Goaldriven attention is required for processing context information (Freedman, Riesenhuber, Poggio, \& Miller, 2003; Hofmann, Schmeichel, \& Baddeley, 2012; Kane, Bleckley, Conway, \& Engle, 2001), and individual instances should be classified according to the corresponding contexts in an implicit manner. A top-down goal due to increased task relevance did not deter the location probability learning in our study, but rather enhanced the specificity of the probability knowledge. These results demonstrate that habitual attention and goal-driven attention are functionally dissociable, as well as interacting functionally to achieve context-specific implicit learning.

To conclude, the present study has shown that multiple sets of statistical knowledge can be learned, depending on the degree of task relevance of the contexts. Though location probability learning is based on habitual attention, this type of incidental learning can be flexible to some extent, when habitual attention functionally interacts with goal-driven attention. Additional research will be needed to further investigate the detailed properties of habitual attention.

Author note This research was supported by the Ministry of Science and ICT and by the National Research Foundation of Korea (NRF-2019R1F1A1062269) and the Korea Brain Research Institute (Grants 19-BR-01-06 and IBS-R001-D12019-b01).
Open practices statement None of the data or materials for the experiments reported here are available, and none of the experiments was preregistered.

\section{References}

Ahn, S., \& Kim, M.-S. (2017, January). Effect of change in target identity on contextual cueing. Talk presented at 54th Annual Meeting of Korean Society for Cognitive \& Biological Psychology, Busan, Korea.

Annac, E., Manginelli, A. A, Pollmann, S., Shi, Z., Müller, H. J., \& Geyer, T. (2013). Memory under pressure: Secondary-task effects on contextual cueing of visual search. Journal of Vision, 13(13), 6: 1-15. https://doi.org/10.1167/13.13.6

Annac, E., Zang, X., Müller, H. J., \& Geyer, T. (2019). A secondary task is not always costly: Context-based guidance of visual search survives interference from a demanding working memory task. British Journal of Psychology, 110, 381-399. https://doi.org/10.1111/bjop.12346

Awh, E., Belopolsky, A. V, \& Theeuwes, J. (2012). Top-down versus bottom-up attentional control: A failed theoretical dichotomy. Trends in Cognitive Sciences, 16, 437-443. https://doi.org/10. 1016/j.tics.2012.06.010

Brainard, D. H. (1997). The Psychophysics Toolbox. Spatial Vision, 10, 433-436. https://doi.org/10.1163/156856897X00357

Carlisle, N. B., Arita, J. T., Pardo, D., \& Woodman, G. F. (2011). Attentional templates in visual working memory. Journal of Neuroscience, 31, 9315-9322. https://doi.org/10.1523/ JNEUROSCI.1097-11.2011

Chun, M. M. (2000). Contextual cueing of visual attention. Trends in Cognitive Sciences, 4, 170-178. https://doi.org/10.1016/S13646613(00)01476-5

Chun, M. M., \& Jiang, Y. (1998). Contextual cueing: Implicit learning and memory of visual context guides spatial attention. Cognitive Psychology, 36, 28-71. https://doi.org/10.1006/cogp.1998.0681

Chun, M. M., \& Jiang, Y. (1999). Top-down attentional guidance on the basis of implicit learning of visual covariation. Psychological Science, 10, 360-365. https://doi.org/10.1111/1467-9280.00168

Crump, M. J. C., Gong, Z., \& Milliken, B. (2006). The context-specific proportion congruent Stroop effect: Location as a contextual cue. Psychonomic Bulletin \& Review, 13, 316-321. https://doi.org/10. 3758/BF03193850

Crump, M. J. C., Vaquero, J. M. M., \& Milliken, B. (2008). Contextspecific learning and control: The roles of awareness, task relevance, and relative salience. Consciousness and Cognition, 17, 22-36. https://doi.org/10.1016/j.concog.2007.01.004

Davis, B., \& Hasson, U. (2018). Predictability of what or in which reduces brain activity, but a bottleneck occurs when both are predictable. NeuroImage, 167, 224-236. https://doi.org/10.1016/j. neuroimage.2016.06.001

De Houwer, J., \& Beckers, T. (2003). Secondary task difficulty modulates forward blocking in human contingency learning. Quarterly Journal of Experimental Psychology, 56B(4), 345-357. https://doi.org/10. 1080/02724990244000296

Druker, M., \& Anderson, B. (2010). Spatial probability aids visual stimulus discrimination. Frontiers in Human Neuroscience, 4, 63:1-10. https://doi.org/10.3389/fnhum.2010.00063

Faul, F., Erdfelder, E., Lang, A.-G., \& Buchner, A. (2007). G*Power 3: A flexible statistical power analysis program for the social, behavioral, and biomedical sceinces. Behavior Research Methods, 39, 175-191. https://doi.org/10.3758/BF03193146

Ferrante, O., Patacca, A., Di Caro, V., Della Libera, C., Santandrea, E., \& Chelazzi, L. (2018). Altering spatial priority maps via statistical learning of target selection and distractor filtering. Cortex, 102, 67-95. https://doi.org/10.1016/j.cortex.2017.09.027 
Freedman, D. J., Riesenhuber, M., Poggio, T., \& Miller, E. K. (2003). A comparison of primate prefrontal and inferior temporal cortices during visual categorization. Journal of Neuroscience, 23, 5235-5246. https://doi.org/10.3410/f.1014765.194583

Frensch, P. A., \& Rünger, D. (2003). Implicit learning. Current Directions in Psychological Science, 12, 13-18. https://doi.org/10.1111/14678721.01213

Geng, J. J., \& Behrmann, M. (2002). Probability cuing of target location facilitates visual search implicitly in normal participants and patients with hemispatial neglect. Psychological Science, 13, 520-525. https://doi.org/10.1111/1467-9280.00491

Geng, J. J., \& Behrmann, M. (2005). Spatial probability as an attentional cue in visual search. Perception \& Psychophysics, 67, 1252-1268. https://doi.org/10.3758/BF03193557

Han, S. W., \& Kim, M.-S. (2009). Do the contents of working memory capture attention? Yes, but cognitive control matters. Journal of Experimental Psychology: Human Perception and Performance, 35, 1292-1302. https://doi.org/10.1037/a0016452

Hofmann, W., Schmeichel, B. J., \& Baddeley, A. D. (2012). Executive functions and self-regulation. Trends in Cognitive Sciences, 16, 174-180. https://doi.org/10.1016/j.tics.2012.01.006

Hübner, R., \& Mishra, S. (2016). Location-specific attentional control is also possible in the Simon task. Psychonomic Bulletin \& Review, 23, 1867-1872. https://doi.org/10.3758/s13423-016-1057-y

JASP Team. (2018). JASP (Version 0.9.1) [Computer software]. Retrieved from https://jasp-stats.org/download/

Jiang, Y. V. (2018. Habitual versus goal-driven attention. Cortex, 102, 107-120. https://doi.org/10.1016/j.cortex.2017.06.018

Jiang, Y., \& Chun, M. M. (2001). Selective attention modulates implicit learning. Quarterly Journal of Experimental Psychology, 54A, 1105-1124. https://doi.org/10.1080/02724980042000516

Jiang, Y. V., Koutstaal, W., \& Twedell, E. L. (2016). Habitual attention in older and young adults. Psychology and Aging, 31, 970-980. https:// doi.org/10.1037/pag0000139

Jiang, Y. V., Sha, L. Z., \& Sisk, C. A. (2018). Experience-guided attention: Uniform and implicit. Attention, Perception, \& Psychophysics, 80, 1647-1653. https://doi.org/10.3758/s13414-018-1585-9

Jiang, Y. V, \& Sisk, C. A. (2019). Habit-like attention. Current Opinion in Psychology, 29, 65-70. https://doi.org/10.1016/j.copsyc.2018.11.014

Jiang, Y., \& Song, J.-H. (2005). Hyperspecificity in visual implicit learning: Learning of spatial layout is contingent on item identity. Journal of Experimental Psychology: Human Perception and Performance, 31, 1439-1448. https://doi.org/10.1037/0096-1523.31.6.1439

Jiang, Y. V., Swallow, K. M., \& Capistrano, C. G. (2013a). Visual search and location probability learning from variable perspectives. Journal of Vision, 13(6), 13:1-13. https://doi.org/10.1167/13.6.13

Jiang, Y. V., Swallow, K. M., \& Rosenbaum, G. M. (2013b). Guidance of spatial attention by incidental learning and endogenous cuing. Journal of Experimental Psychology: Human Perception and Performance, 39, 285-297. https://doi.org/10.1037/a0028022

Jiang, Y. V., Swallow, K. M., Rosenbaum, G. M., \& Herzig, C. (2013c). Rapid acquisition but slow extinction of an attentional bias in space. Journal of Experimental Psychology: Human Perception and Performance, 39, 87-99. https://doi.org/10.1037/a0027611

Jiang, Y. V., Swallow, K. M., \& Sun, L. (2014a). Egocentric coding of space for incidentally learned attention: Effects of scene context and task instructions. Journal of Experimental Psychology: Learning Memory and Cognition, 40, 233-250. https://doi.org/10.1037/a0033870

Jiang, Y. V., Swallow, K. M., Won, B.-Y., Cistera, J. D., \& Rosenbaum, G. M. (2015). Task specificity of attention training: The case of probability cuing. Attention, Perception, \& Psychophysics, 77, 50-66. https://doi.org/10.3758/s13414-014-0747-7

Jiang, Y. V., Won, B.-Y., \& Swallow, K. M. (2014b). First saccadic eye movement reveals persistent attentional guidance by implicit learning. Journal of Experimental Psychology: Human Perception and Performance, 40, 1161-1173. https://doi.org/10.1037/a0035961
Kane, M. J., Bleckley, M. K., Conway, A. R. A., \& Engle, R. W. (2001). A controlled-attention view of working-memory capacity. Journal of Experimental Psychology: General, 130, 169-183. https://doi. org/10.1037/0096-3445.130.2.169

Kim, J.-H., \& Kim, M.-S. (2016, October). The effect of frequency change on the contextual cueing effect. Poster presented at Annual Fall Conference of Korean Society for Emotion \& Sensibility, Busan, Korea.

Lavie, N. (1995). Perceptual load as a necessary condition for selective attention. Journal of Experimental Psychology: Human Perception and Performance, 21, 451-468. https://doi.org/10.1037/0096-1523. 21.3.451

Lavie, N. (2005). Distracted and confused? Selective attention under load. Trends in Cognitive Sciences, 9, 75-82. https://doi.org/10. 1016/j.tics.2004.12.004

Oh, S.-H., \& Kim, M.-S. (2004). The role of spatial working memory in visual search efficiency. Psychonomic Bulletin \& Review, 11, 275281. https://doi.org/10.3758/BF03196570

Olson, I. R., \& Chun, M. M. (2002). Perceptual constraints on implicit learning of spatial context. Visual Cognition, 9, 273-302. https://doi. org/10.1080/13506280042000162

Pelli, D. G. (1997). The VideoToolbox software for visual psychophysics: Transforming numbers into movies. Spatial Vision, 10, 437-442. https://doi.org/10.1163/156856897X00366

Rosenbaum, G. M., \& Jiang, Y. V. (2013). Interaction between scene-based and array-based contextual cuing. Attention, Perception, \& Psychophysics, 75, 888-899. https://doi.org/10.3758/s13414-0130446-9

Salovich, N. A., Remington, R. W., \& Jiang, Y. V. (2018). Acquisition of habitual visual attention and transfer to related tasks. Psychonomic Bulletin \& Review, 25, 1052-1058. https://doi.org/10.3758/s13423017-1341-5

Schwark, J., \& Dolgov, I. (2013). The influence of spatial and feature probability cuing in visual search. Perception, 42, 470-472. https:// doi.org/10.1068/p7469

Soto, D., Heinke, D., Humphreys, G. W., \& Blanco, M. J. (2005). Early, involuntary top-down guidance of attention from working memory. Journal of Experimental Psychology: Human Perception and Performance, 31, 248-261. https://doi.org/10.1037/0096-1523.31.2.248

Summerfield, J. J., Lepsien, J., Gitelman, D. R., Mesulam, M. M., \& Nobre, A. C. (2006). Orienting attention on the basis of long-term memory experience. Neuron, 49, 905-916. https://doi.org/10.1016/ j.neuron.2006.01.021

Vickery, T. J., Sussman, R. S., \& Jiang, Y. V. (2010). Spartial context learning survives interference from WM load. Journal of Experimental Psychology: Human Perception and Performance, 36, 1358-1371. https://doi.org/10.1037/a0020558.

Wagenmakers, E.-J., Love, J., Marsman, M., Jamil, T., Ly, A., Verhagen, J., ... Morey, R. D. (2018a). Bayesian inference for psychology. Part II: Example applications with JASP. Psychonomic Bulletin \& Review, 25, 58-76. https://doi.org/10. 3758/s13423-017-1323-7

Wagenmakers, E.-J., Marsman, M., Jamil, T., Ly, A., Verhagen, J., Love, J., .. Morey, R. D. (2018b). Bayesian inference for psychology: Part I. Theoretical advantages and practical ramifications. Psychonomic Bulletin \& Review, 25, 35-57. https://doi.org/10. 3758/s13423-017-1343-3

Wang, Y., Song, Y., Qu, Z., \& Ding, Y. (2010). Task difficulty modulates electrophysiological correlates of perceptual learning. International Journal of Psychophysiology, 75, 234-240. https://doi.org/10.1016/ j.ijpsycho.2009.11.006

Wendt, M., Kluwe, R. H., \& Vietze, I. (2008). Location-specific versus hemisphere-specific adaptation of processing selectivity. Psychonomic Bulletin \& Review, 15, 135-140. https://doi. org/10.3758/PBR.15.1.135

Won, B.-Y., \& Jiang, Y. V. (2015). Spatial working memory interferes with explicit, but not probabilistic cuing of spatial attention. Journal 
of Experimental Psychology: Learning, Memory, and Cognition, 41, 787-806. https://doi.org/10.1037/xlm0000040

Won, B.-Y., Lee, H. J., \& Jiang, Y. V. (2015). Statistical learning modulates the direction of the first head movement in a largescale search task. Attention, Perception, \& Psychophysics, 77, 2229-2239. https://doi.org/10.3758/s13414-015-0957-7
Woodman, G. F., \& Luck, S. J. (2004). Visual search is slowed when visuospatial working memory is occupied. Psychonomic Bulletin \& Review, 11, 269-274. https://doi.org/10.3758/BF03196569

Publisher's note Springer Nature remains neutral with regard to jurisdictional claims in published maps and institutional affiliations. 